\title{
Air mass source determines airborne microbial diversity at the ocean-atmosphere interface of the Great Barrier Reef marine ecosystem
}

\author{
Stephen D. J. Archer ${ }^{1,2} \cdot$ Kevin C. Lee $\mathbb{C}^{2} \cdot$ Tancredi Caruso $^{3} \cdot$ Katie King-Miaow ${ }^{2} \cdot$ Mike Harvey $^{4}$ - Danwei Huang ${ }^{5} \cdot$ \\ Benjamin J. Wainwright ${ }^{5}$ Stephen B. Pointing $\mathbb{( i )}^{1,5,6}$
}

Received: 31 August 2019 / Accepted: 4 November 2019 / Published online: 21 November 2019

(c) The Author(s) 2019. This article is published with open access

\begin{abstract}
The atmosphere is the least understood biome on Earth despite its critical role as a microbial transport medium. The influence of surface cover on composition of airborne microbial communities above marine systems is unclear. Here we report evidence for a dynamic microbial presence at the ocean-atmosphere interface of a major marine ecosystem, the Great Barrier Reef, and identify that recent air mass trajectory over an oceanic or continental surface associated with observed shifts in airborne bacterial and fungal diversity. Relative abundance of shared taxa between air and coral microbiomes varied between 2.2 and $8.8 \%$ and included those identified as part of the core coral microbiome. We propose that this variable source of atmospheric inputs may in part contribute to the diverse and transient nature of the coral microbiome.
\end{abstract}

Airborne microbial transport is central to dispersal outcomes [1] and several studies have demonstrated diverse microbial biosignatures are recoverable from the atmosphere. Microbial transport has been shown to occur across inter-continental distances above terrestrial habitats [2-4].

These authors contributed equally: Stephen D. J. Archer, Kevin C. Lee

Supplementary information The online version of this article (https:// doi.org/10.1038/s41396-019-0555-0) contains supplementary material, which is available to authorized users.

Stephen B. Pointing

stephen.pointing@yale-nus.edu.sg

1 Yale-NUS College, National University of Singapore, Singapore 138527, Singapore

2 Institute for Applied Ecology New Zealand, Auckland University of Technology, Auckland 1142, New Zealand

3 School of Biological Sciences and Institute for Global Food Security, Queen's University Belfast, Belfast BT9 7BL Northern Ireland, UK

4 National Institute of Water and Atmospheric Research (NIWA), Wellington 6021, New Zealand

5 Department of Biological Sciences, National University of Singapore, Singapore 117558, Singapore

6 Institute of Nature and Environmental Technology, Kanazawa University, Kanazawa 920-1151, Japan
Variation has been recorded seasonally [5, 6], with underlying land use [7], and due to stochastic weather events such as dust storms [8]. Above marine systems, the abundance of microorganisms decreases exponentially with distance from land [9], but relatively little is known about potential patterns in biodiversity for airborne microorganisms above the oceans. Here we test the hypothesis that persistent microbial inputs to the ocean-atmosphere interface of the Great Barrier Reef ecosystem vary according to surface cover (i.e. land vs. ocean) during transit of the air-mass.

The Great Barrier Reef is an ideal model system for research on bio-aerosols because incoming air mass during the average residence time for microorganisms in air [10] arises from two distinct sources: a terrestrial continental source in Australia transported across east and northeast dust paths and an oceanic source in the Coral Sea (Fig. 1a). Our study took advantage of a persistent flat calm sea state during September-October 2016 (www.marineweather.net. $\mathrm{au}$ ). This minimised interference from microorganisms that are aerosolised by marine spray in heavier sea states at the Great Barrier Reef [11]. We recovered massive bulk-phase air samples at $25 \mathrm{~m}$ above sea level using a high-volume liquid impinger apparatus (Coriolis $\mu$, Bertin Technologies, France) [12] during a voyage of the RV Investigator to circumnavigate the reef (Supplementary Information, Supplementary Methods). We used the National Oceanic and Atmospheric Administration (NOAA) HYSPLIT-WEB 
Fig. 1 a HYSPLIT back trajectory analysis for modelled transit routes (3 days residence time [12]) with colours

representing air mass origin over continent (green) or ocean (red) surface, scale bar $=500 \mathrm{~km}$;

Rarefaction curves for samples by origin for (b) bacteria (continent $n=8$, Ocean $n=19$ ) and (c) fungi (continent $n=8$, ocean $n=17$ ). Chao1 diversity index by origin for (d) bacteria and (e) fungi. Individual data points are shown as circles, boxplot whiskers represent 1.5 times the interquartile range from the first to third quartiles; f Heatmap for bacterial amplicon sequence variants (ASVs) that explain $95 \%$ of observed diversity by origin with control samples included (continent $n=$ 8 , ocean $n=19$, control $n=7$ ); g Heatmap for fungal ASVs that explain $95 \%$ of observed diversity by origin with control samples included (continent $n=$ 8 , ocean $n=17$, control $n=5$ ). All samples were sequenced to near asymptote. More detailed heatmaps that incorporate the 1000 most abundant ASVs for bacteria and fungi are shown in Supplementary Information, Fig. S3 a.

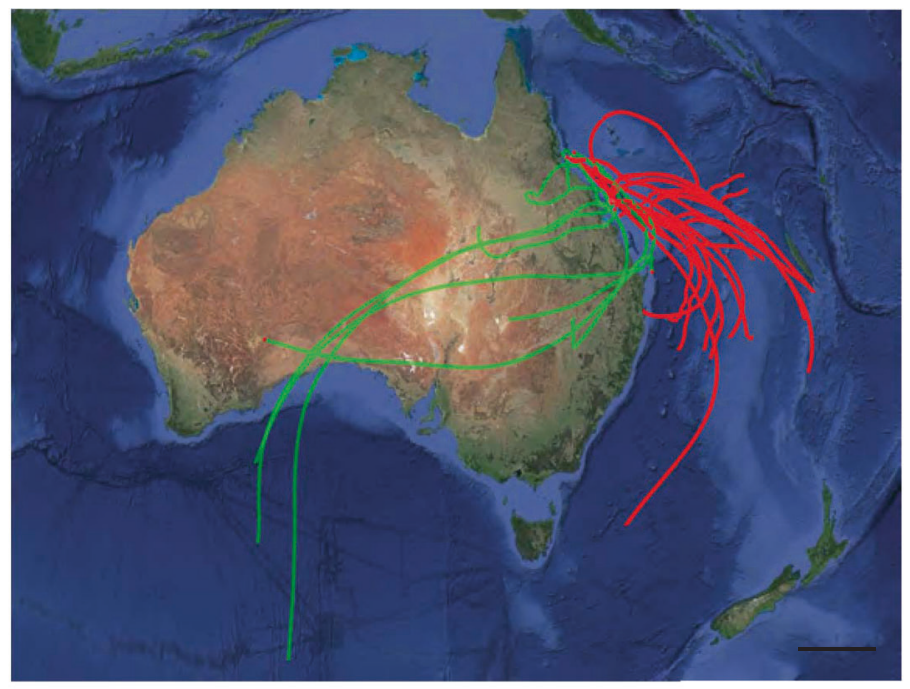

b.

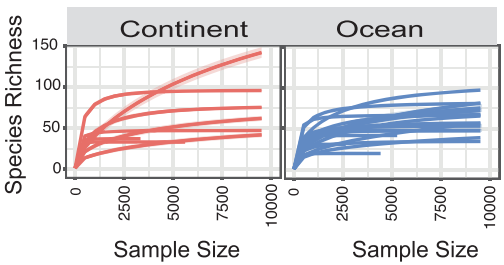

C.

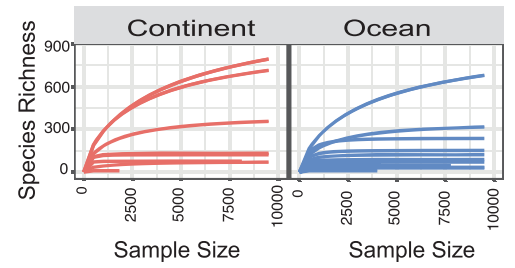

d.

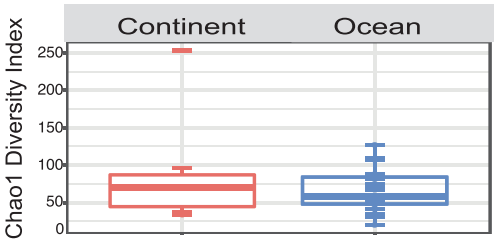

e.

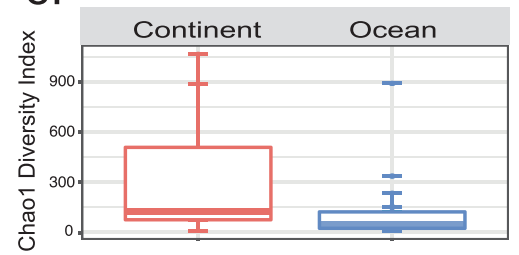

f.

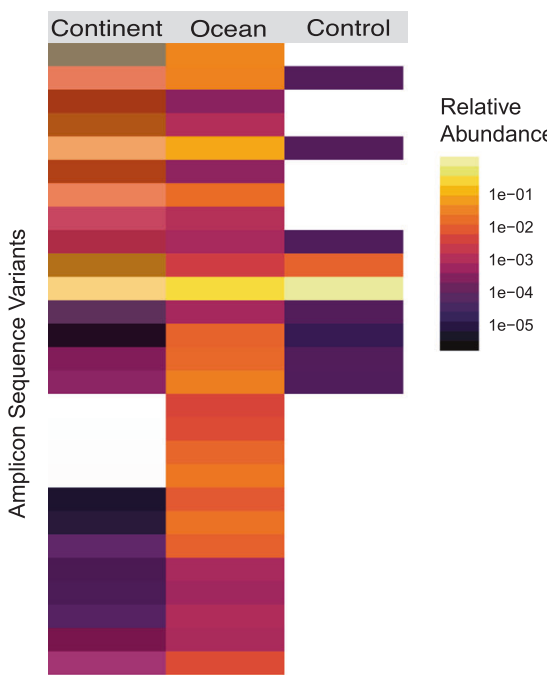

g

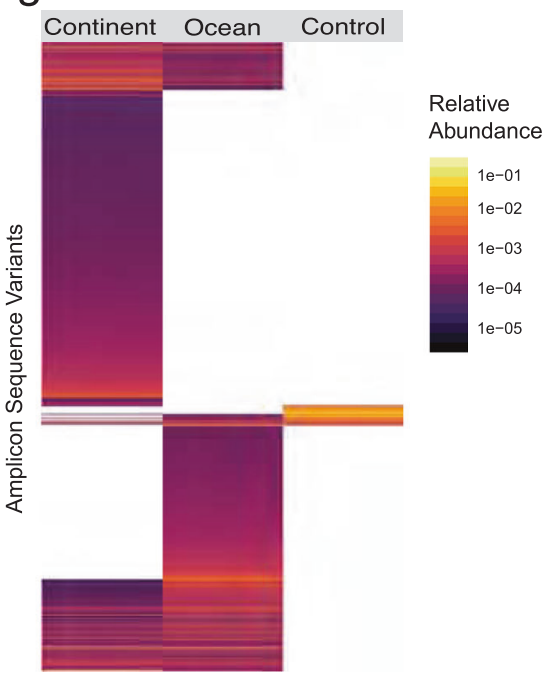

model (https://ready.arl.noaa.gov/HYSPLIT.php) to identify back trajectories of air mass during the average residence time for microbial cells in air [10]. Back trajectories for air mass could be delineated clearly into those with recent transit over either continental Australia (continental path) or the Coral Sea (oceanic path) (Fig. 1a; 
Supplementary Information, Fig. S1). The concurrent concentration of atmospheric radon gas measured in real time was consistently higher from back trajectories originating over continental Australia compared with those that originated from the ocean (Mann-Whitney U Test, $P=0.003$ ). This measurement further validated the binning of air mass into a continental or oceanic origin.

Consistent with predictions that microbial biomass is extremely low and unpredictable over the oceans, we achieved recoverable DNA from 27 of 53 bulk-phase air samples (mean recovery $0.23 \mathrm{ng} / \mathrm{m}^{3}$, STDEV $0.21 \mathrm{ng} / \mathrm{m}^{3}$; Supplementary Information, Table S1) with no significant difference in DNA yield between sample groups (MannWhitney $\mathrm{U}$ Test, $P=0.37$ ). High-throughput sequencing of the bacterial $16 \mathrm{~S}$ rRNA gene and fungal Internal Transcribed Spacer region were performed as previously described [12] and phylogenetic analysis of amplicon sequence variants (ASVs) were used to estimate diversity [13] (Supplementary Information, Supplementary Methods). Sequencing of control samples revealed very low recovery of putative contaminant microbial signatures. A total of only 17 out of 1403 bacterial and 5 out of 3775 fungal sequences were statistically classified as putative contaminants (Supplementary Information, Supplementary Methods). Most samples were sequenced to near-asymptote (Supplementary Information, Figs. 1b, c).

Transit above oceanic or continental surfaces were significantly correlated with bacterial and fungal community structures at the ocean-atmosphere interface above the Great Barrier Reef (bacteria: PERMANOVA $R^{2}=0.07233$, pseudo $F=1.9494, P=0.024$; manyglm $L R T=1650, P=$ 0.02 ; fungi PERMANOVA $R^{2}=0.08413$, pseudo $F=$ 2.1125, $P=0.044$, manyglm $L R T=6033, P=0.034$ ). Kendall's rank correlation tau identified a weak but significant distance relationship (bacteria $0.19 P=<0.001$ and fungi $0.10 P=0.008$ ) (Fig. S5), which suggests air source but also distance can affect community similarities. In terms of overall taxa richness, bacteria displayed similar richness but higher phylogenetic diversity in continental vs. ocean-derived samples (Fig. 1d; Supplementary Information Fig. S2), and similarly the fungi displayed markedly greater richness in continent-derived samples (Fig. 1e). Heatmaps of the most abundant ASVs representing 95\% of overall diversity illustrate the major taxonomic differences in bacterial (Fig. 1f) and fungal (Fig. 1g) assemblages between ocean and continent-derived air mass. Repeating this analysis with the 1,000 most abundant taxa from each meta-library captured $99.98 \%$ of bacterial and $84.24 \%$ of fungal diversity in the study and yielded similar results (Supplementary Information Fig. S3). Principal coordinate analysis using Bray-Curtis dissimilarities of bacterial and fungal communities revealed somewhat mixed trends in ordination plots (Supplementary Information Fig. S4); however, changes in relative abundance at the phylum level were striking between days when air mass was sourced from continent or ocean (Fig. 2c, e).

Overall, the shared ASVs between samples from ocean and continental air masses represented a large proportion of total bacterial (87.9 and $89.6 \%$ ) and fungal (64.9 and 69.1\%) reads. However, the abundances for dominant genera were highly variable between air masses. For example, the three most abundant bacterial genera Burkholderia, Ralstonia and Sphingomonas were present at 28, 14.4 and 5.5\% in oceanic air and 37, 0.5 and 20\% in continental air masses while the three most abundant fungal genera (Alternaria, Cladosporium and Mycosphaerella) were present at 6.4, 4.5 and $5.3 \%$ in oceanic air and 13.5, 3.5 and $1.4 \%$ in continental air masses, respectively. A recent study characterising bacteria aerosolized from a seawater mesocosm concluded $\sim 75 \%$ of taxa were alpha, beta and gamma Proteobacteria [14], and notably identified Bradyrhizobium and Ralstonia as highly abundant taxa thus establishing a potential marine source for some of the most abundant airborne taxa in our study. While the core ASVs represented a high proportion of reads, many less abundant ASVs were specific to either oceanic (538 bacteria, 1335 fungi) or continental (395 bacteria, 1810 fungi) air masses (Fig. 1f, g).

Shifts in relative abundance for bacteria mainly occurred within the phylum Proteobacteria where the dominant genus recovered changed with air mass sampled among Acinetobacter, Alistipes, Bradyrhizobium and Ralstonia (Fig. 2a, c). All have been recovered as isolates or environmental rRNA gene sequences from both marine and terrestrial sources, thus making any attempt at source tracking challenging. For the fungi terrestrial air sources supported higher diversity than marine sources and this reflected the abundance of terrestrial fungal habitats. A striking observation was that $56 \%$ of recovered genera supported yeast-like taxa, and this provides support for a marine origin since oceanic waters are thought to support elevated abundance of yeasts over filamentous fungi [15]. Shifts in diversity were less pronounced overall for fungi (Fig. 2b, d), and were partially obscured by the high diversity relative to bacteria at the genus level. Major shifts in relative abundance occurred for Aureobasidium, Cladosporium, Coprinopsis, Rhodosporidiobolus and Rhodotorula, and all of these genera have known terrestrial and marine records, although it should be noted that terrestrial fungal spores have been recorded in many marine microbial diversity assessments but are unlikely to be active components of an ocean surface water microbiome.

We further interrogated the phylogenetic diversity of continental and oceanic air masses using Net Relatedness Index (NRI) analysis to estimate the level of phylogenetic structuring and putative recruitment from local (i.e. ocean or continental source only) and regional (all sources) pools 


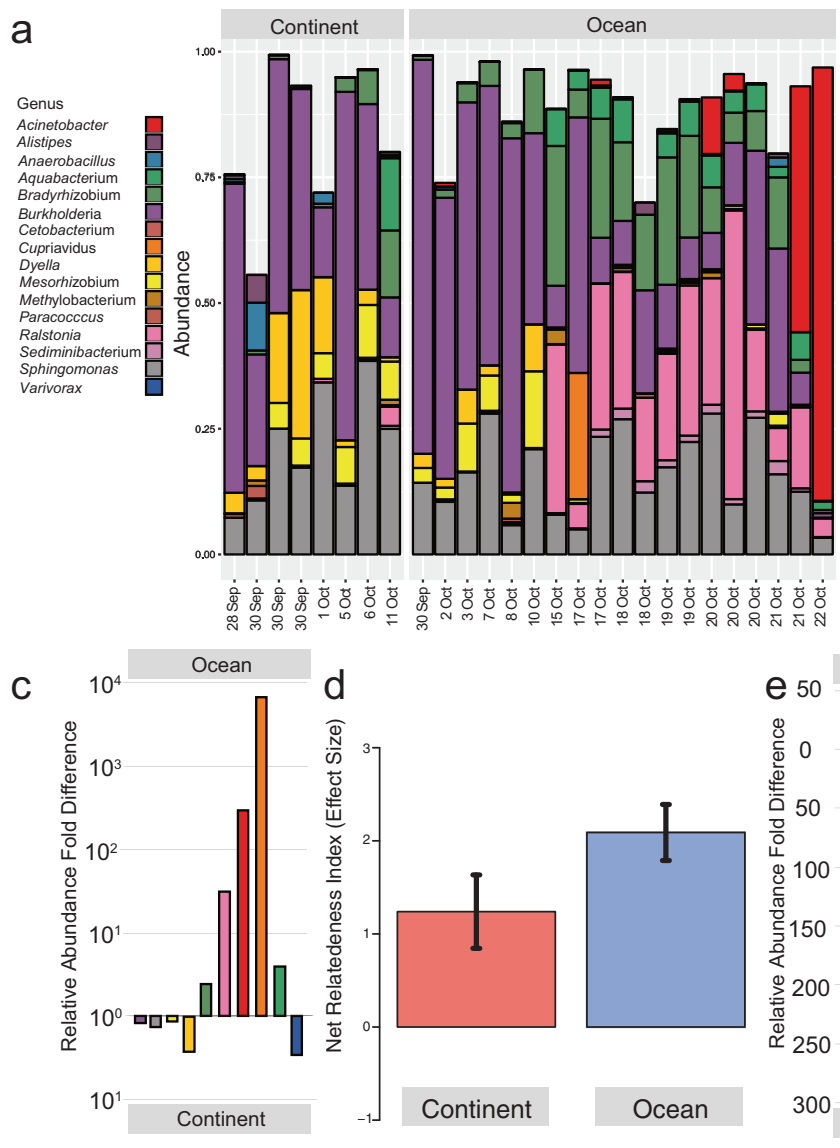

Fig. 2 Relative abundance and genus level taxonomic assignment for ASV of all taxa with $>0.5 \%$ mean relative abundance for (a) bacteria and (b) fungi; Relative abundance fold differences for taxa from ocean and continent-derived air mass, for (c) bacteria and (e) fungi; Net Relatedness Index (NRI) of phylogenetic structure for continental and ocean-derived atmosphere taxa for (d) bacteria (continent $n=8$, ocean $n=19$, control $n=7$ ) and (f) fungal (continent $n=8$, ocean $n=17$, control $n=5$ ) ASVs, error bars indicate standard error of the mean for

[12]. This analysis revealed that bacterial assemblages from both oceanic and continental origin displayed positive NRI values with effect sizes indicative of non-random assembly (Fig. 2d, f). Communities were thus phylogenetically highly structured, which is possibly due to environmental filtering of traits during transit over the different surface covers. The fungi from continental sources displayed a similar though more variable trend of phylogenetic structuring although in ocean-derived samples this clustering was relatively weak indicating they were more randomly assembled. Overall our analyses support the hypothesis that long-range transport of microbial taxa in air results in differential recruitment and selection during transit over oceanic or continental surfaces $[12,16,17]$.

It has been proposed that airborne deposition of microorganisms may be a source of symbionts and pathogenic taxa to coral reefs $[18,19]$. In order to establish the potential
Continent Ocean

Genus Alternaria Botrytis Cladosporium Cladosporium Coprinellus Coprinellus
Coprinopsis Coprinopsis Curvularia Didymella Epicoccum Filobasidium Hansfordia Itersonilia Malassezia Mycosphaer
Nigrospora Nigrospora
Peniophora Peniophora
Periconia Phaeococcor Phaeococcomy
Phanerochaete Phlebiopsis Preussia Pseudopithomyces Rhodosporidiobol Rhodotorula Trametes

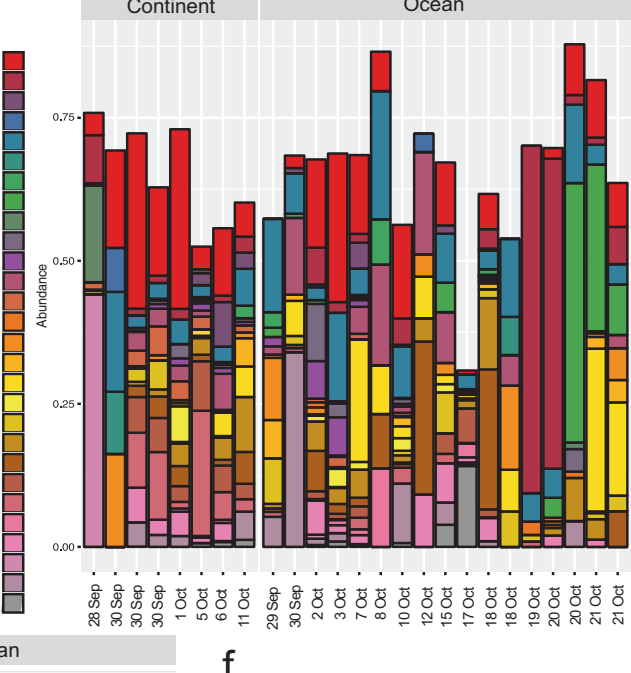

Ocean f

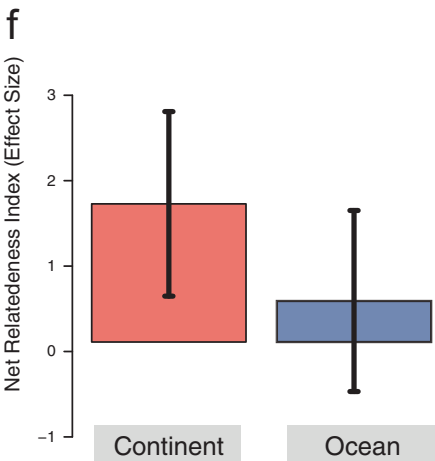

all samples. Bacterial analysis was based on a phylogenetic tree derived using maximum likelihood analysis of 16S rRNA gene ASVs. Fungal analysis utilised a neighbour joining tree that was compared with topology of whole genome trees for fungi at high taxonomic ranks, in order to be conservative regarding the phylogenetic information provided by ITS (Supplementary Information, Supplementary Methods)

recruitment of airborne microorganisms to coral reefs we compared our sequence data to that obtained for coral microbiomes. We identified only one study with directly comparable sequence data (Supplementary Information, Supplementary Methods) recovered from Porites lutea coral microbiomes in the Andaman Sea and Gulf of Thailand [20]. A total of $6.7 \%$ coral reads shared the same ASVs as our airborne bacterial dataset. The air masses from oceanic sources had higher relative abundance of ASVs in common with the coral dataset $(8.8 \%)$ compared to air with recent continental transit (2.2\%) (Supplementary Information, Fig. S6a). Three of the six shared genera (Bradyrhizobium, Burkholderia and Sediminibacterium) were identified as contributing to the core coral microbiome for the Andaman Sea and Gulf of Thailand [20]. The class Burkholdariales that supports Burkholdaria has been identified as a source of ubiquitous coral endosymbionts globally [21]. Relaxing 
the stringency of this comparison to the genus level (i.e. $\geq 97 \%$ sequence matches) to account for possible biogeographic variation and ecological variation resulted in higher relative abundance of shared taxa between the datasets. Using this criteria $36 \%$ coral bacterial taxa were shared with the atmospheric microbiome, and $77 \%$ of oceanic and $79 \%$ continental derived atmospheric taxa matched those of the coral microbiome (Supplementary Information, Fig. S6b).

We also compared our bacterial ASV sequences with a recent study of tropical corals from the Singapore Straits, South China Sea [22], which shared significant bacterial 16 S rRNA sequence overlaps (Supplementary Information, Supplementary Methods). We identified (with $\geq 97 \%$ sequence identities) $6.5 \%$ of coral taxa were shared with the atmospheric microbiome, and $94.4 \%$ of ocean-derived air and $87.8 \%$ of continent-derived air taxa were shared with the coral microbiome (Supplementary Information, Fig. S6c). Finally, we broadly screened taxa from our sequence libraries at taxon level (genus) to known coralassociated taxa including putative symbionts and pathogens identified using different rRNA loci or approaches (e.g. DGGE and Sanger sequencing) (Supplementary Information, Supplementary Methods). Using this approach we estimated the ocean-derived air mass supported $8.4 \%$ bacterial genera that may include coral associates whilst for continental sources this value was lower at 2.9\% (Supplementary Information, Table S2). The most differentially abundant fungal taxon from this comparison (Ralstonia sp.) showed greatest sequence similarity to a previously identified coral endosymbiont (Supplementary Information, Table S3) thus indicating any atmosphere-coral microbiome connectivity may be highly variable. For our fungal library there were few coral-associated fungal sequences with which to make comparisons and little phylogenetic information, but at a broad level we identified $8.7 \%$ of oceanderived taxa and $6.2 \%$ continent-derived taxa that have recorded coral associates in the same genus (Supplementary Information, Table S3).

Overall our study has provided a unique insight on the variability of airborne microbial communities above the largest coral reef ecosystem on Earth and yielded clues that atmospheric, oceanic and terrestrial biomes may be interconnected via the atmospheric microbiome. Our study indicates that the view of coral microbiomes as harbouring few long term residents and instead comprising largely a "diverse transient community that is responsive to surrounding environment" [23] may be explained in part by the variability in airborne microbial diversity above reefs that may act as recruitment reservoirs. Improved understanding of cross-biome microbial biocomplexity and interaction will require coordinated and directly comparable research effort from atmospheric, marine and terrestrial microbiologists.

\section{Data availability}

All sequence data generated by this study has been submitted to the EMBL European Nucleotide Archive (ENL) under BioProject PRJEB31630 with sample accession numbers ERS3215240 to ERS3215312.

Acknowledgements Collaboration in this work was supported through ARC Discovery Project (grant number DP150101649) and NIWA's Research Programme in Ocean-Climate Interactions (grant number 2017/18 SCI). Financial support for laboratory research was provided by the Yale-NUS College Start-up Fund. Author SDJA was supported by a Postdoctoral Fellowship from Auckland University of Technology (AUT). We thank ARC Discovery Project Principal Investigators Prof Zoran Ristovski and Luke Cravigan (Queensland University of Technology), the officers and crew of the RV Investigator (IN2016_V05), and Tony Bromley and Sally Grey (NIWA) for their outstanding logistical support and Timothy Lawrence (AUT) for his DNA sequencing support. Radon data was provided by Scott Chambers and Alastair Williams (Australian Nuclear Science and Technology Organisation).

Author contribution SDJA, KCL, TC, MH and SBP conceived the study; SDJA conducted ship-board fieldwork; SDJA and KCL performed laboratory experiments; SDJA, KCL, TC, KK-M, BJW and SBP performed data analysis; SDJA, MH, DH, BJW and SBP analysed and interpreted the findings; SBP wrote the manuscript.

\section{Compliance with ethical standards}

Conflict of interest The authors declare that they have no conflict of interest.

Publisher's note Springer Nature remains neutral with regard to jurisdictional claims in published maps and institutional affiliations.

Open Access This article is licensed under a Creative Commons Attribution 4.0 International License, which permits use, sharing, adaptation, distribution and reproduction in any medium or format, as long as you give appropriate credit to the original author(s) and the source, provide a link to the Creative Commons license, and indicate if changes were made. The images or other third party material in this article are included in the article's Creative Commons license, unless indicated otherwise in a credit line to the material. If material is not included in the article's Creative Commons license and your intended use is not permitted by statutory regulation or exceeds the permitted use, you will need to obtain permission directly from the copyright holder. To view a copy of this license, visit http://creativecommons. org/licenses/by/4.0/.

\section{References}

1. Hanson CA, Fuhrman JA, Horner-Devine MC, Martiny JBH. Beyond biogeographic patterns: processes shaping the microbial landscape. Nat Rev Microbiol. 2012;10:497-506.

2. Barberán A, Henley J, Fierer N, Casamayor EO. Structure, interannual recurrence, and global-scale connectivity of airborne microbial communities. Sci Total Environ. 2014;487:187-95.

3. Spracklen DV, Heald CL. The contribution of fungal spores and bacteria to regional and global aerosol number and ice nucleation immersion freezing rates. Atmos Chem Phys. 2014;14:9051-9. 
4. Caliz J, Triadó-Margarit X, Camarero L, Casamayor EO. A longterm survey unveils consistent seasonal trends in the airborne microbiome composition coupled to air masses circulation. Proc Natl Acad Sci USA. 2019;115:12229-34.

5. Woo AC, Brar MS, Chan Y, Lau MCY, Leung FCC, Scott JA, et al. Temporal variation in airborne microbial populations and microbially-derived allergens in a tropical urban landscape. Atmos Environ. 2013;74:291-300.

6. Bowers RM, McCubbin IB, Hallar AG, Fierer N. Seasonal variability in airborne bacterial communities at a high-elevation site. Atmos Environ. 2012;50:41-9.

7. Bowers R, McLetchie S, Knight R, Fierer N. Spatial variability in airborne bacterial communities across land-use types and their relationship to the bacterial communities of potential source environments. ISME J. 2011;5:601-12.

8. Favet J, Lapanje A, Giongo A, Kennedy S, Aung Y-Y, Cattaneo A, et al. Microbial hitchhikers on intercontinental dust: catching a lift in Chad. ISME J. 2013;7:850-67.

9. Mayol E, Arrieta JM, Jiménez MA, Martínez-Asensio A, GarciasBonet N, Dachs J, et al. Long-range transport of airborne microbes over the global tropical and subtropical ocean. Nat Commun. 2017;8:201.

10. Burrows SM, Butler T, Jöckel P, Tost H, Kerkweg A, Pöschl U, et al. Bacteria in the global atmosphere-Part 2: modeling of emissions and transport between different ecosystems. Atmos Chem Phys. 2009;9:9281-97.

11. Cropp R, Gabric A, van Tran D, Jones G, Swan H, Butler H. Coral reef aerosol emissions in response to irradiance stress in the Great Barrier Reef, Australia. Ambio. 2018;47:671-81.

12. Archer S, Lee K, Caruso T, Lee C, Maki T, Cowan D, et al. Microbial dispersal limitation to isolated soil microbial habitats of Antarctica. Nat Microbiol. 2019;4:925-32.

13. Callahan BJ, McMurdie PJ, Holmes SP. Exact sequence variants should replace operational taxonomic units in marker-gene data analysis. ISME J. 2017;11:2639-43.
14. Michaud JM, Thompson LR, Kaul D, Espinoza JL, Richter RA, $\mathrm{Xu} \mathrm{ZZ}$, et al. Taxon-specific aerosolization of bacteria and viruses in an experimental ocean-atmosphere mesocosm. Nat Commun. 2018;9:2017.

15. Kutty SN, Philip R. Marine yeasts-a review. Yeast. 2008;25:465-83.

16. Mayol E, Jiménez MA, Herndl GJ, Duarte CM, Arrieta JM. Resolving the abundance and air-sea fluxes of airborne microorganisms in the North Atlantic Ocean. Front Microbiol. 2014;5:557.

17. Šantl-Temkiv T, Gosewinkel U, Starnawski P, Lever M, Finster K. Aeolian dispersal of bacteria in southwest Greenland: their sources, abundance, diversity and physiological states. FEMS Microbiol Ecol. 2018;94:fiy031.

18. Weir-Brush JR, Garrison VH, Smith GW, Shinn EA. The relationship between gorgonian coral (cnidaria: gorgonacea) diseases and african dust storms. Aerobiologia. 2004;20:119-26.

19. Rosenberg E, Koren O, Reshef L, Efrony R, Zilber-Rosenberg I. The role of microorganisms in coral health, disease and evolution. Nat Rev Microbiol. 2007;5:355-62.

20. Pootakham W, Mhuantong W, Yoocha T, Putchim L, Sonthirod C, Naktang C, et al. High resolution profiling of coral-associated bacterial communities using full-length $16 \mathrm{~S}$ rRNA sequence data from PacBio SMRT sequencing system. Sci Rep. 2017;7:2774.

21. Ainsworth TD, Krause L, Bridge T, Torda G, Raina JB, Zakrzewski M, et al. The coral core microbiome identifies rare bacterial taxa as ubiquitous endosymbionts. ISME J. 2015;9:2261-74.

22. Wainwright BJ, Afiq-Rosli L, Zahn GL, Huang D. Characterisation of coral-associated bacterial communities in an urbanised marine environment shows strong divergence over small geographic scales. Coral Reefs. 2019. https://doi.org/10.1007/s00338019-01837-1.

23. Leggat W, Ainsworth TD, Herrera C, Bongaerts P, HernandezAgreda A. Rethinking the coral microbiome: simplicity exists within a diverse microbial biosphere. MBio. 2018;9:1-14. 